\title{
Validation of the Risk Score of the Mortality and Lower Limb Loss Considering Ambulatory Status after Surgical Revascularization in Maintaining Patients with Dialysis
}

\author{
Haruto Yamazaki, RPT, ${ }^{1}$ Hisae Hayashi, RPT, PhD, ${ }^{2}$ Morio Kawamura, MD, PhD, ${ }^{3}$ \\ Ayana Sasaki, RPT, ${ }^{4}$ Eriko Kondo, RPT, ${ }^{5}$ Shinya Ito, RPT, ${ }^{6}$ and Kenji Wakai, MD, $\mathrm{PhD}^{7}$
}

\begin{abstract}
Surgical revascularization is performed to preserve limb and to maintain functional status of patients with critical limb ischemia (CLI). The PREVENT III risk score helps to predict the postoperative course of CLI. However, this score is not available to estimate the risk of amputation or death properly in patients with hemodialysis (HD) and tissue loss (HD: 4 points, Tissue loss: 3 points), because they are classified as a high-risk group. Therefore, we investigated 213 patients with revascularized HD for CLI and proposed prognosis amputation or death for patients with HD risk score (PAD for HD risk score). PAD for HD risk score (nonambulation: 3 points, ulcer/gangrene: 2 points, GNRI<92: 2 points, CRP $>0.3 \mathrm{mg} / \mathrm{dl}$ : 1 point, Age $\geq 75$ : 1 point) is more accurate for the prediction of amputation or death than the PREVENT III risk score (area under the curve [AUC]: 0.79 [95\% confidence interval: $\mathrm{Cl}: 0.71-0.87], p<0.01$ vs. AUC: 0.63 [95\% $\mathrm{Cl}: 0.56-0.71])$. The patients were stratified into three groups by total score in ascending order. The rate of 1-year amputation-free survival and independent ambula-
\end{abstract}

${ }^{1}$ Department of Rehabilitation, Kohno Clinical Medicine Research Institute, Shinagawa Rehabilitation Hospital, Tokyo, Japan

${ }^{2}$ The Faculty of Rehabilitation and Care, Seijoh University, Tokai, Aichi, Japan

${ }^{3}$ Department of Physical, College of Life and Health Sciences, Chubu University, Kasugai, Aichi, Japan

${ }^{4}$ Department of Rehabilitation, Municipal Kisogawa Hospital, Ichinomiya, Aichi, Japan

${ }^{5}$ Department of Rehabilitation, Nagoya Kyoritsu Hospital, Nagoya, Aichi, Japan

${ }^{6}$ Department of Rehabilitation, Karatsu Red Cross Hospital, Karatsu, Saga, Japan

${ }^{7}$ Department of Preventive Medicine, Nagoya University Graduate School of Medicine, Nagoya, Aichi, Japan

Received: June 11, 2017; Accepted: June 11, 2017

Corresponding author: Hisae Hayashi, RPT, PhD. The Faculty of Rehabilitation and Care, Seijoh University, 2-172 Fukinodai, Tokai, Aichi 476-0014, Japan

Tel: +81-52-601-6000, Fax: +81-52-601-6010

E-mail: hisaehayashi@seijoh-u.ac.jp

This is a translation of J Jpn Coll Angiol 2016; 56: 85-91. tory status were significantly different among three groups. PAD for HD risk score is useful for rehabilitation planning in patients with HD and CLI. (This is a translation of J Jpn Coll Angiol 2016; 56: 85-91.)

Keywords: dialysis, critical limb ischemia, surgical revascularization, risk score, multivariate analysis

\section{Introduction}

Rehabilitation care is increasingly performed after surgical revascularization (SR) of lower limbs to prevent amputation and maintain function. Several studies reported that, even after successful revascularization, critical limb ischemia (CLI) led to limb loss in one out of four patients within 5 years. ${ }^{1-3)}$ It is necessary to establish an appropriate rehabilitation goal considering the likelihood of preserving the patient's lower limbs and improved life expectancy. It is possible to stratify the risk of death/lower-limb amputation after SR using a conventional scoring system (PREVENT III risk score). ${ }^{4)}$ This scoring system is useful for setting rehabilitation goals and planning a program. However, in the PREVENT III risk score, maximum points are allotted to dialysis dependence. Because most dialysis dependent patients were assigned to a high-risk group, the stratification of the risk of death/lower-limb amputation in hemodialysis (HD) patients using this score was limited. Therefore, we propose an alternative risk score predicting prognosis of amputation or death (PAD) for HD patient risk score. To investigate predictive power of this score, we considered the ambulation status ${ }^{5,6)}$ as a factor that may influence the lower limbs/functional prognosis after SR as well as chronic inflammation/malnutrition, ${ }^{7)}$ which were reported to influence the prognosis of HD patients. 


\section{Patients and Methods}

\section{Patients and study setting}

A retrospective survey of electronic medical records was conducted in patients on maintenance HD who were admitted to Nagoya Kyoritsu Hospital and who underwent lower extremity SR for ischemic pain at rest or ischemic ulcers between April 2005 and November 2011. We excluded patients diagnosed with malignant tumors, those who died within one week after SR (perioperative period), those who simultaneously underwent revascularization and major amputation, and those who had undergone previous major amputation.

\section{Data collection}

We collected data regarding baseline patient demographics (age, sex, height, and body weight), complicating conditions (hypertension, hyperlipidemia, diabetes, and coronary artery disease), and clinical indicators (ulcers and/or gangrene) at the time of surgery. In addition, we collected data on laboratory parameters (serum albumin level, CRP level, hematocrit, and platelet count) within 1 month prior to SR, ambulatory status 2 weeks after $\mathrm{SR}$, and the date of death/lower-limb amputation. We set the endpoint as death/lower-limb amputation within 1 year after SR. Nutritional status was assessed using the Geriatric Nutritional Risk Index (GNRI); a GNRI of $<92$ was considered malnourished. ${ }^{8)}$ The ambulation status was evaluated using the "movement" parameter of the Functional Independence Measure (FIM). ${ }^{9)}$ Patients with a score of $\geq 6$ FIM were regarded as independent and those with a score of $\leq 5$ were regarded as dependent.

\section{Statistical analysis}

Statistical analysis with Cox's proportional hazard model was performed to find variables associated with death/ lower-limb amputation. Variables with a p-value $\leq 0.1$ on univariate analysis were then included in a multivariate analysis (forced entry method). The risk score was ex- pressed as an integral number by dividing the $\beta$-coefficient of the predictor by 0.3 and rounding off to the nearest integer value in accordance with the previous study $\left.{ }^{4}\right)$ Furthermore, area under the curve (AUC) was calculated based on the ROC curves of the PAD for HD risk score proposed in this study and PREVENT III risk score, and the predictive power was compared between the two scores. In addition, patients were divided into three groups (0-3, 4-6, and 7-9 points groups) based on the total risk score, and 1-year amputation-free survival (AFS) and ambulation rates were calculated. The AFS rate was calculated using the KaplanMeier method. Significance was tested using the log-rank test and multiple comparisons with Bonferroni's method. A p-value of 0.05 was regarded as significant.

\section{Ethical considerations}

The protocol of this study was approved by the Ethics Review Board of Nagoya University (Approval No.: 12-502) and Clinical Research Committee of Nagoya Kyoritsu Hospital. The information of only those patients who had consented comprehensively for the study was analyzed. Furthermore, the database used in this study was anonymized in a linkable fashion, and only the researchers could access the identification number.

\section{Results}

Of 256 patients (318 limbs) who underwent SR between April 2005 and November 2011, 213 (272 limbs) with a median follow up of 575 days were analyzed. Patients who could not be followed up were excluded (Fig. 1). The characteristics of the limbs are shown in Table 1. Overall, 20 patients (21 limbs) underwent amputations and 35 patients (38 limbs) died within 1 year.

The results of uni- and multivariate analyses using death/limb loss after 1 year as a dependent variable are presented in Table 2. Variables with a p-value of $\leq 0.1$ in the univariate analysis consisted of age $\geq 75$ years, GNRI $<92$, CRP level $>0.3 \mathrm{mg} / \mathrm{dl}$, hematocrit $\leq 30 \%$, non-

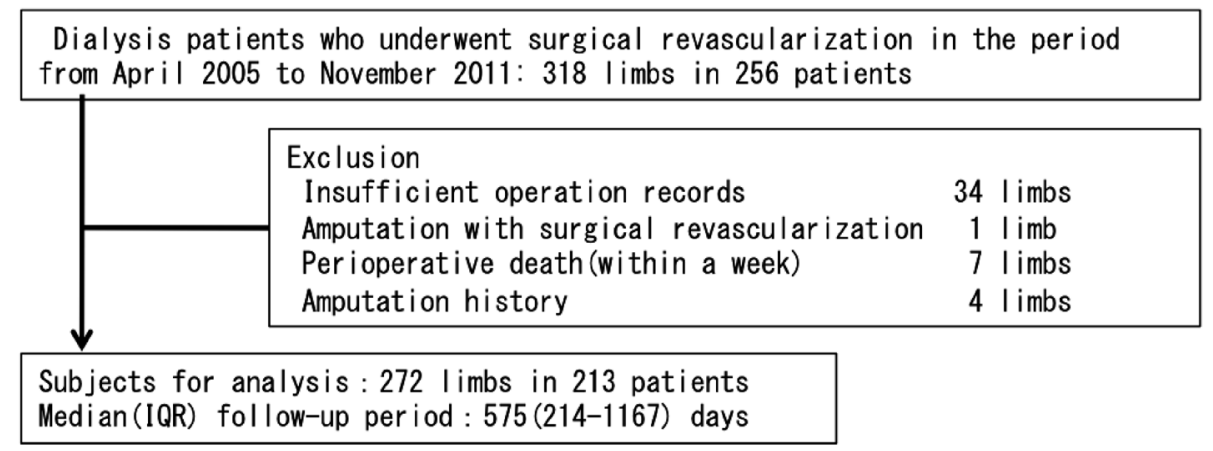

IQR; Interquartile range

Fig. 1 Selection process of target patients and limbs. 
ambulation, and ulcer formation/necrosis. Variables with a p-value of $<0.05$ in the multivariate analysis consisted of postoperative ambulatory status and GNRI. We calculated the PAD for HD risk scores of five variables. We included three variables, namely, ulcer formation/necrosis, ${ }^{4)}$ CRP, ${ }^{3)}$ and age, $\left.{ }^{4}\right)$ which were indicative of independent risk factors for the AFS rate, and the two variables detected on multivariate analysis. The scores of postoperative ambulation status, GNRI, ulcer formation/necrosis, CRP, and age were 3, 2, 2, 1, and 1 point, respectively (Table 2 ). Concerning a hematocrit level of $\leq 30 \%, 4$ ) the sign of the regression coefficient was reversed on multivariate analy-

Table 1 Characteristics of the target limbs $(n=272)$

\begin{tabular}{|c|c|}
\hline Characteristics & \\
\hline Sex; Male, n (\%) & $195(71.7)$ \\
\hline Age (years), mean $\pm S D$ & $66.0 \pm 9.1$ \\
\hline$\geqq 75, \mathrm{n}(\%)$ & $52(19.1)$ \\
\hline $\mathrm{BMI}\left[\mathrm{kg} / \mathrm{m}^{2}\right]$, mean $\pm \mathrm{SD}$ & $21.3 \pm 3.5$ \\
\hline Hypertension, n (\%) & $153(56.3)$ \\
\hline Hypercholesterolemia, n (\%) & $55(20.2)$ \\
\hline Diabetes, n (\%) & $153(56.3)$ \\
\hline Coronary artery disease, $\mathrm{n}(\%)$ & $249(91.5)$ \\
\hline Ulcer/Gangrene, n (\%) & $148(54.4)$ \\
\hline Ambulation status, $\mathrm{n}(\%)$ & $152(55.9)$ \\
\hline Serum albumin $(\mathrm{g} / \mathrm{dl})$, mean $\pm \mathrm{SD}$ & $3.4 \pm 0.5$ \\
\hline GNRI, mean \pm SD & $88.9 \pm 8.6$ \\
\hline$<92, \mathrm{n}(\%)$ & $110(40.4)$ \\
\hline $\mathrm{CRP}[\mathrm{mg} / \mathrm{dl}]$, mean $\pm \mathrm{SD}$ & $2.60 \pm 4.6$ \\
\hline$>0.3 \mathrm{mg} / \mathrm{dl}, \mathrm{n}(\%)$ & $155(57.0)$ \\
\hline Hematocrit $(\%)$, mean \pm SD & $32.4 \pm 5.6$ \\
\hline$<30 \%, \mathrm{n}(\%)$ & $79(29.0)$ \\
\hline Platelet $\left[\times 10^{4} / \mu \mathrm{l}\right]$, mean \pm SD & $22.5 \pm 8.9$ \\
\hline
\end{tabular}

SD: standard deviation; BMI: body mass index; GNRI: geriatric nutritional risk index sis; therefore, this factor was excluded from the prediction model.

The AUC of the PREVENT III and PAD for HD risk scores was $0.63(95 \% \mathrm{CI}: 0.56-0.71, \mathrm{p}=0.02)$ and 0.79 (95\% CI: $0.71-0.87, \mathrm{p}<0.01)$, respectively; there was a significant difference in the predictive capacity between the two scores $(\mathrm{p}<0.01)$ (Fig. 2). The overall AFS rate was $79.0 \%$ (95\% CI: 71.8-84.8). Based on the total risk score, patients were divided into three groups (0-3, 4-6, and 7-9 points groups), and the AFS and independent ambulation rates 1 year after SR were compared. There were significant differences among the groups (Fig. 3 ).

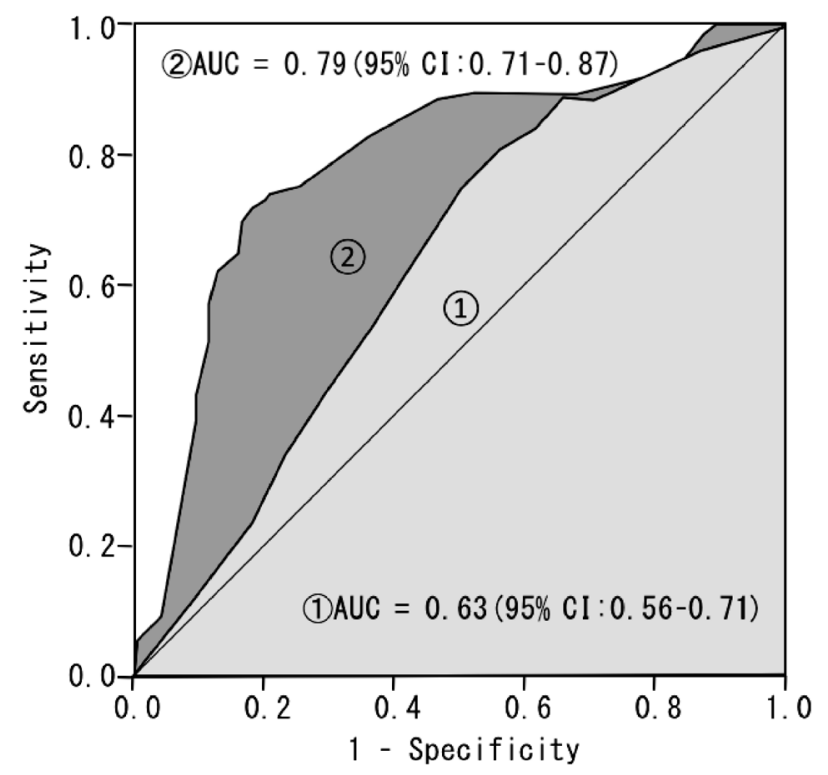

Fig. 2 ROC curve by (1)PREVENT III risk score and (2)PAD for $\mathrm{HD}$ risk score.

Table 2 Univariate and a multivariate hazard ratios for death or amputation of limb

\begin{tabular}{|c|c|c|c|c|c|}
\hline & $\begin{array}{c}\text { Death or } \\
\text { Amputation n (\%) }\end{array}$ & $\begin{array}{c}\text { Univariate } \\
\text { HR }(95 \% \mathrm{Cl}) \mathrm{p} \text {-value }\end{array}$ & $\begin{array}{c}\text { Multivariate } \\
\mathrm{HR}(95 \% \mathrm{Cl}) \mathrm{p} \text {-value }\end{array}$ & $\begin{array}{l}\text { Regression } \\
\text { coefficient }\end{array}$ & Score \\
\hline Age $\geqq 75$ years & 10/53 (18.9) & $1.51(0.94-2.45) p=0.09$ & $1.49(0.74-2.98) p=0.27$ & 0.40 & 1 \\
\hline Male & 41/188 (21.8) & $0.99(0.65-1.50) p=0.99$ & - & - & - \\
\hline Hypertension & $31 / 149(20.8)$ & $0.97(0.66-1.43) p=0.89$ & - & - & - \\
\hline Hypercholesterolemia & $14 / 53(26.4)$ & $1.31(0.82-2.10) p=0.26$ & - & - & - \\
\hline Diabetes & $14 / 53(21.6)$ & $1.19(0.81-1.77) p=0.37$ & - & - & - \\
\hline Coronary artery disease & $54 / 249(21.7)$ & $1.04(0.55-1.94) p=0.91$ & - & - & - \\
\hline Non-ambulation & 45/129 (34.9) & $3.80(2.52-5.74) p<0.01$ & $2.42(1.30-4.51) p<0.01$ & 0.88 & 3 \\
\hline Ulcer/Gangrene & 40/143 (28.0) & $2.19(1.45-3.31) p<0.01$ & $1.68(0.93-3.05) p=0.09$ & 0.52 & 2 \\
\hline GNRI $<92$ & $30 / 117(25.6)$ & $2.63(1.52-4.56) p=0.01$ & $1.91(1.08-3.39) p=0.03$ & 0.65 & 2 \\
\hline $\mathrm{CRP}>0.3 \mathrm{mg} / \mathrm{dl}$ & 43/155 (27.7) & $2.71(1.68-4.38) p<0.01$ & $1.49(0.79-2.80) p=0.22$ & 0.40 & 1 \\
\hline Hematocrit $\leqq 30 \%$ & $19 / 78(24.4)$ & $1.54(1.02-2.32) p=0.04$ & * & - & - \\
\hline Platelet & - & $1.01(0.98-1.03) p=0.64$ & - & - & - \\
\hline
\end{tabular}

*Hematocrit was excluded from the variables due to a negative regression coefficient in the multivariate analysis. HR: hazard ratio; GNRI: geriatric nutritional risk index; $\mathrm{Cl}$ : confidence interval 


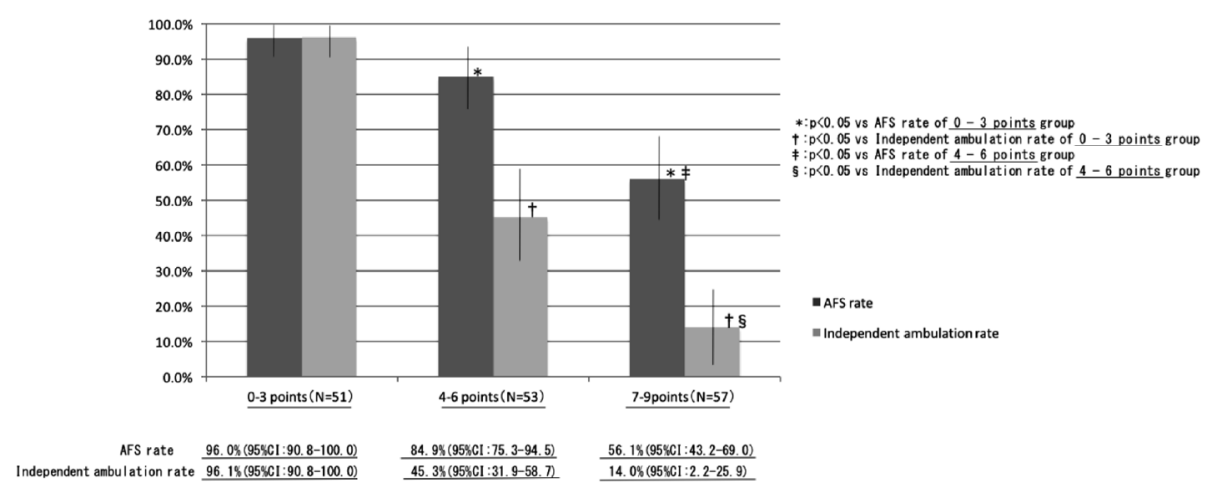

Fig. 3 One year amputation-free survival rate and independent ambulation rate. The patients were divided in to three groups by assessing PAD for HD risk score (0-3 points $n=51$, 4-6 points $n=53,7-9$ points $n=57$ ). One year amputation-free survival rates and independent ambulation rates were significantly different among three groups.

\section{Discussion}

In this study, we proposed a risk score (PAD for HD risk score) to predict the prognosis of HD patients after lowerlimb artery revascularization and compared its predictive power. It was confirmed that the predictive power of the PAD for HD risk score (AUC: 0.79) was significantly higher than that of the PREVENT III risk score (AUC: 0.63). Furthermore, patients were divided into three groups based on the total score, and the AFS and ambulation rates 1 year after SR was compared. There were significant differences among the groups. These results suggest that the PAD for HD risk score proposed in this study is useful for setting rehabilitation goals, considering the AFS rate of HD patients.

The PAD for HD risk score comprises five items: age, the ambulation status as an index of functional prognosis, CRP as a variable for chronic inflammation, GNRI that indicates nutritional status, and ulcer formation/necrosis that is specific to the disease. Several studies reported that ambulation status was associated with the prognosis of lower limbs. ${ }^{5,6,10)}$ They also indicated the difficulty in improving the postoperative ambulation status of patients who were not ambulated preoperatively. ${ }^{5)}$ In this study, the postoperative ambulation status was used as an explanatory variable because it may reflect lower-limb function after the complete or partial improvement of lower-limb ischemia due to SR. Our results support previous studies, $5,6,10)$ that is, the ambulation status was associated with the prognosis of lower limbs and life expectancy. Furthermore, one study indicated that in CLI patients, the characteristics of those with non-ambulation status included the presence of several underlying diseases, such as hypertension, hyperlipidemia, heart disease, diabetes, and renal failure..$^{5)}$ Ambulation status may reflect their general health status.

According to another study, the AFS rate 1 year after surgery in HD patients was markedly lower than that in non-HD patients. ${ }^{4)}$ Some studies associated this result with chronic inflammation or malnutrition. ${ }^{7,10)}$ Our study also showed similar findings. In our study, the mean GNRI was $88.9 \pm 8.6$ and the percentage of patients with a GNRI of $<92$ was $40.4 \%$, confirming malnutrition in most patients. Based on these results, the nutritional status must be considered when setting the rehabilitation goal of HD patients after SR.

Our results show that the AFS rate in the group with the lowest risk score (0-3 points) was $96.0 \%$ and that the ambulation rate was $96.1 \%$. These results suggest that physical therapy for this group should focus on walking training. Furthermore, patients with a PAD for HD risk score of $0-3$ points were assigned to the medium (AFS rate: $73.0 \%$ ) or high (AFS rate: $44.6 \%$ ) risk groups based on the PREVENT III risk score. However, the observed outcome was often different from that predicted by the PREVENT III risk score. Stratification could be performed by considering disease-specific variable and/or lower-limb function of HD patients after surgery in whom the risk of death/lower-limb amputation is reportedly high. On the other hand, the AFS rate in the group with the highest risk score (7-9 points) was $56.1 \%$ whereas the ambulation rate was $14.0 \%$. This result suggests that patients for whom walking training is not indicated account for a specific subgroup of patients with high-risk scores. After SR, activities of daily living (ADL) tend to become more dependent on others. ${ }^{11)}$ Another study indicated that patients who are unable to expect improvement in ambulatory status may enjoy better quality of life through pain relief or foot ulcer healing. ${ }^{5}$ For this reason, we propose that these patients should receive a physical therapy program, involving exercises to maintain articular function and muscle strength and promote peripheral blood flow, and training in daily activities to improve ADL.

In this study, the AFS rate in the group with the highest risk score was $56.1 \%$, higher than in a previous study) 
(44.6\%). It was possibly because the prognosis of HD patients and limb salvage rate in Japan were more favorable than those in the United States ${ }^{12,13)}$ and because the ulceration rate of this study $(54.4 \%)$ differed from the previous study $\left.{ }^{4}\right)(73.6 \%)$. The difference in the incidence of ulcers may have been related to regular screening for the prevention of lower-limb amputation and appropriate revascularization before ulcer/gangrene progression in the patients in our study. ${ }^{14)}$

\section{Limitations of This Study}

This study has several inherent limitations. Firstly, because it was conducted at a single center, the usefulness of this score should be investigated in a larger number of institutions in future studies. Secondly, it was a retrospective survey and lacked accurate information about graft failure and hemodynamics of the superficial skin $\left(\mathrm{TcPO}_{2}\right.$ and SPP) for some patients, making it impossible to review the two parameters as explanatory variables. One study reported that graft occlusion after revascularization led to lower-limb loss. ${ }^{15)}$ Regarding superficial skin blood flow, the other study indicated that hypoperfusion increased the risk of lower-limb loss ${ }^{16}$; therefore, it may be necessary to establish a risk score including the two parameters as explanatory variables.

\section{Conclusion}

The proposed PAD for HD risk score has an acceptable predictive value of AFS 1 year after surgery in HDdependent patients. The results demonstrated that predictive power was improved by including the influence of the postoperative ambulatory status and malnutrition that may be specific to HD patients. The PAD for HD risk score may contribute to set appropriate goals of rehabilitation based on the accurate prediction of prognosis in HD-dependent patients.

\section{Acknowledgments}

The authors would like to thank the vascular surgeons and physical therapists of Nagoya Kyoritsu Hospital for their cooperation in completing this study.

\section{Disclosure Statement}

There is no conflict of interest to be disclosed with respect to this article.

\section{References}

1) Arvela E, Söderström M, Korhonen M, et al. Finnvasc score and modified Prevent III score predict long-term outcome after infrainguinal surgical and endovascular revascularization for critical limb ischemia. J Vasc Surg 2010; 52: 121825.

2) Söderström M, Arvela E, Aho PS, et al. High leg salvage rate after infrainguinal bypass surgery for ischemic tissue loss (Fontaine IV) is compromised by the short life expectancy. Scand J Surg 2010; 99: 230-4.

3) Ishii H, Kumada Y, Toriyama T, et al. Prognostic values of C-reactive protein levels on clinical outcome after endovascular therapy in hemodialysis patients with peripheral artery disease. J Vasc Surg 2010; 52: 854-9.

4) Schanzer A, Mega J, Meadows J, et al. Risk stratification in critical limb ischemia: derivation and validation of a model to predict amputation-free survival using multicenter surgical outcomes data. J Vasc Surg 2008; 48: 1464-71.

5) Flu HC, Lardenoye JH, Veen EJ, et al. Functional status as a prognostic factor for primary revascularization for critical limb ischemia. J Vasc Surg 2010; 51: 360-71. e1.

6) Rollins KE, Coughlin PA. Functional outcomes following revascularisation for critical limb ischaemia. Eur J Vasc Endovasc Surg 2012; 43: 420-5.

7) Stenvinkel P, Heimbürger O, Paultre F, et al. Strong association between malnutrition, inflammation, and atherosclerosis in chronic renal failure. Kidney Int 1999; 55: 1899-911.

8) Bouillanne O, Morineau G, Dupont C, et al. Geriatric Nutritional Risk Index: a new index for evaluating at-risk elderly medical patients. Am J Clin Nutr 2005; 82: 777-83.

9) Granger CV, Hamilton BB, Keith RA, et al. Advances in functional assessment for medical rehabilitation. Top Geriatr Rehabil 1986; 1: 59-74.

10) Logar CM, Pappas LM, Ramkumar N, et al. Surgical revascularization versus amputation for peripheral vascular disease in dialysis patients: a cohort study. BMC Nephrol 2005; 6: 3.

11) Cieri E, Lenti M, De Rango P, et al. Functional ability in patients with critical limb ischaemia is unaffected by successful revascularisation. Eur J Vasc Endovasc Surg 2011; 41: 25663.

12) Rajagopalan S, Dellegrottaglie S, Furniss AL, et al. Peripheral arterial disease in patients with end-stage renal disease observations from the dialysis outcomes and practice patterns study (DOPPS). Circulation 2006; 114: 1914-22.

13) Combe C, Albert JM, Bragg-Gresham JL, et al. The burden of amputation among hemodialysis patients in the dialysis outcomes and practice patterns study (DOPPS). Am J Kidney Dis 2009; 54: 680-92.

14) Kumada Y, Furuhashi K, Sugimoto M, et al. Long-term outcome of lower extremities bypass surgery in hemodialysis patients. J Jpn Coll Angiol 2006; 46: 681-7.

15) Goodney PP, Nolan BW, Schanzer A, et al.; Vascular Study Group of Northern New England. Factors associated with amputation or graft occlusion one year after lower extremity bypass in Northern New England. Ann Vasc Surg 2010; 24 : 57-68.

16) Hatakeyama S, Saito M, Ishigaki K, et al. Skin perfusion pressure is a prognostic factor in hemodialysis patient. Int $\mathrm{J}$ Nephrol 2012; Article ID 385274: 7 pages. 